\title{
Efecto de la suplementación de ácidos grasos omega 3 de cadena corta en el metabolismo de lípidos del cerebro y su relación con el índice de encefalización en ratas lactantes
}

\section{Effect of short chain omega 3 fatty acids supplementation on brain lipids metabolism and their relationship with the encephalization quotient in lactating rats}

\author{
Ma. del Consuelo Figueroa-García ${ }^{1}$, Raymundo Coate-Camacho² y Ricardo Mejía-Zepeda2* \\ ${ }^{1}$ Investigación independiente, Ciudad de México; '2Laboratorio 4, Unidad de Biomedicina, Facultad de Estudios Superiores Iztacala, Universidad \\ Nacional Autónoma de México, Tlalnepantla, Edo. de México. México
}

\begin{abstract}
Resumen
Introducción: Los ácidos grasos omega 3 (AG(3) están ganando popularidad en el tratamiento y prevención de enfermedades asociadas al metabolismo de los carbohidratos y como coadyuvantes en el desarrollo de las capacidades cognitivas, por lo que es común escuchar que se deben consumir desde la infancia, sin tomar en cuenta la poca información cientifica que se tiene para respaldar estas afirmaciones. Objetivo: Analizar los efectos de los $A G \omega 3$ en ratas durante el periodo de lactancia. Material y métodos: Se utilizaron 24 ratas Wistar recién nacidas, que se dividieron en dos grupos (machos y hembras). De cada grupo, 6 ratas recibieron una dosis de $125 \mathrm{mg} / \mathrm{kg} / \mathrm{día}$ de ácido alfa linolénico $(A G \omega 3 C C)$. Al destete de los animales se midieron los parámetros fisiológicos (PF), y fueron sacrificados para la obtención de muestras. Se extrajeron mitocondrias hepáticas para determinar la concentración de malondialdehído (MDA), se hizo control respiratorio (CR), y se determinó la composición de ácidos grasos de tejido del cerebro y del hígado. Resultados: En el grupo con suplemento de AG(3)CC se observan los $P F$ alterados, el $C R$ y MDA ligeramente aumentados, sin cambios en los parámetros morfométricos del cerebro y el índice de encefalización (EQ).

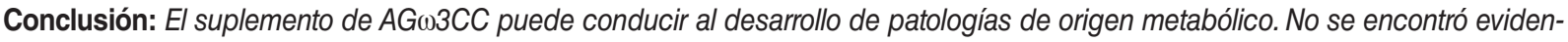
cia de que el consumo de $A G \omega 3 C C$ tenga un efecto benéfico en el desarrollo del cerebro o que conduzca al incremento del EQ.
\end{abstract}

Palabras clave: Rata lactante. Ácidos grasos omega 3. Índice de encefalización.

\begin{abstract}
Introduction: The omega 3 fatty acids ( $\omega 3 F A)$ are earning popularity against the treatment and prevention of diseases associated to the carbohydrates metabolism, as well as adjuvants for the development of cognitive capabilities, because of that they are recommended to be consumed since childhood but without taking into account the few scientific information for supporting such statements. Objective: To analyze the effects of the $\omega 3$ FA in rats during the lactation. Material and methods: 24 newborn Wistar rats were divided in two groups (males and females). Six animals from each group received a daily dose of $125 \mathrm{mg} / \mathrm{kg}$ of the short chain $\omega 3$ fatty acid (SC $\omega 3$ FA) alfa linolenic acid. At the end of nursing of the animals the physiological parameters $(P P)$ were measured and they were sacrificed for obtaining the samples. The hepatic mitochondria were isolated for obtaining the malondialdehyde (MDA) concentration, respiratory control $(R C)$ and it was determined the fatty
\end{abstract}

Correspondencia:

*Ricardo Mejía-Zepeda

E-mail: rmejia@unam.mx
Fecha de recepción: 19-02-2020

Fecha de aceptación: 05-03-2020

DOI: 10.24875/RHJM.20000034
Disponible en internet: 19-06-2020 Rev Hosp Jua Mex. 2020;87(2):94-105

www.revistahospitaljuarez.com 1405-9622/○ 2020 Sociedad Médico-Quirúrgica del Hospital Juárez de México, A.C. Publicado por Permanyer. Este es un artículo open access bajo la licencia CC BY-NC-ND (http://creativecommons.org/licenses/by-nc-nd/4.0/). 
acids (FA) composition of the brain and liver. Results: In the group with supplement of SC $\omega 3$ FA it was found altered PP, and slightly augmented RC, and MDA; no changes in morphometric parameters of brain and the Encephalization Quotient (EQ). Conclusion: The supplement of SC $\omega 3$ FA can drive to the development of pathologies of metabolic origin. It was not found evidence that the intake of SC 13 FA has beneficial effects in the development of the brain nor in the EQ.

Key words: Lactating rats. Omega 3 fatty acids. Encephalization Quotient.

\section{Introducción}

Uno de los factores de mayor impacto en el desarrollo de los animales, incluido el ser humano, es la nutrición, la cual ha cambiado a lo largo de la historia ${ }^{1}$, ya sea por factores sociales, ambientales o derivados del estudio y el desarrollo tecnológico de nuevas materias primas utilizables como alimentos o complementos alimenticios. Además de servir como alimento, muchas moléculas se han utilizado como nutracéuticos o elementos terapéuticos en la cura, tratamiento o prevención del desarrollo de diversas enfermedades ${ }^{2}$. Estos productos generalmente se obtienen a partir de alimentos y se comercializan como concentrados (píldoras, polvos, homogenizados u otros) de algún compuesto, hipotéticamente bioactivo, dentro de una matriz (alimenticia o no $)^{3}$, en cantidades que exceden a las obtenidas de los alimentos en forma natural. Un aspecto social de suma importancia es que estos productos no requieren un registro sanitario de la Comisión Federal Contra Riesgos Sanitarios (COFEPRIS) para su comercialización. Los fabricantes y/o distribuidores únicamente necesitan el llamado «aviso de funcionamiento", por lo que su venta no requiere de receta médica. El descubrimiento de la importancia del consumo de los ácidos grasos, y sobre todo de los llamados esenciales, ha significado desde 1929 un arma de doble filo, pues si bien es cierto que la carencia de estas moléculas potencialmente causa daño, el consumo excesivo puede provocar alteración del metabolismo y, a largo plazo, es decir, de forma crónica, llevar al individuo a padecer enfermedades. Dentro de los suplementos alimenticios se encuentran los ácidos grasos omega 3 (AG $(03)$, a los cuales se les atribuyen efectos benéficos en el tratamiento y prevención de padecimientos de tipo crónico-degenerativo, como diabetes, cáncer, obesidad, hipertensión o trastornos cardiovasculares, además de retrasar el envejecimiento ${ }^{2,4}$, sin tener pruebas científicas contundentes. Además de lo indicado anteriormente, los AG $\omega 3$ se han estudiado como factores del desarrollo cognitivo durante la etapa infantil, y derivado de ello se ofertan como una promesa para el incremento de las capacidades intelectuales en los niños. Es obligado señalar que la promesa de una mejora de la inteligencia deriva únicamente de estudios de tipo clínico ${ }^{5}$, en los cuales no se integra evidencia fisiológica, anatómica o bioquímica que explique los efectos psicosociales y cognitivos encontrados. Es importante reconocer que entre los grupos vulnerables o a los que se recomienda el consumo crónico o no regulado de estas sustancias están: los bebés prematuros, niños con problemas de adaptación o déficit de atención, las personas de la tercera edad, mujeres en gestación, pacientes con problemas cardíacos, cáncer 0 enfermedades crónico-degenerativas, como diabetes y síndrome metabólico, o pacientes psiquiátricos ${ }^{6}$. El presente estudio tiene como finalidad analizar los efectos derivados de la suplementación de AG $\omega 3$ de cadena

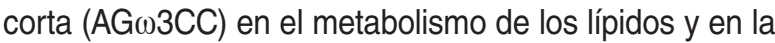
función mitocondrial del hígado y del cerebro de ratas en etapa de lactación, así como la evaluación de los parámetros fisiológicos que orientan en el estudio del índice de encefalización de los individuos y sus posibles efectos en el potencial cognitivo.

\section{Material y métodos}

Se utilizaron 24 ratas Wistar lactantes (0 a 30 días), y se formaron cuatro grupos $(n=6)$ : machos control (MCN), machos con tratamiento (M $(\omega)$ ), hembras control $(\mathrm{HCN})$ y hembras con tratamiento $(\mathrm{H} \omega 3)$. El tratamiento consistió en la administración diaria de $125 \mathrm{mg} /$ $\mathrm{kg}$ de peso corporal de aceite de linaza como fuente de ácido linolénico (ALA), el cual es considerado un AG $\omega 3 C C$, por vía oral, durante 30 días. La dosis utilizada se calculó con base en la dosis recomendada para humanos (31.25 mg/kg de peso) y el factor metabólico de la especie ${ }^{7}$. El peso de los animales se midió todos los días durante el tratamiento con la finalidad de ajustar la dosis de aceite de linaza. Al término del tratamiento se evaluó el perfil fisiológico (concentración de glucosa, colesterol y triglicéridos en sangre y curva de tolerancia a la glucosa [CTG]) con tiras reactivas y un equipo Acutrend de Roche@. Para la realización de este estudio se siguieron las recomendaciones de la Norma Oficial Mexicana (NOM 062-ZOO-1999) ${ }^{8}$. Al término del tratamiento, los animales se anestesiaron con pentobarbital sódico $40 \mathrm{mg} / \mathrm{kg}$ de peso, intraperitoneal. Se realizó exsanguinación por punción cardíaca (con 


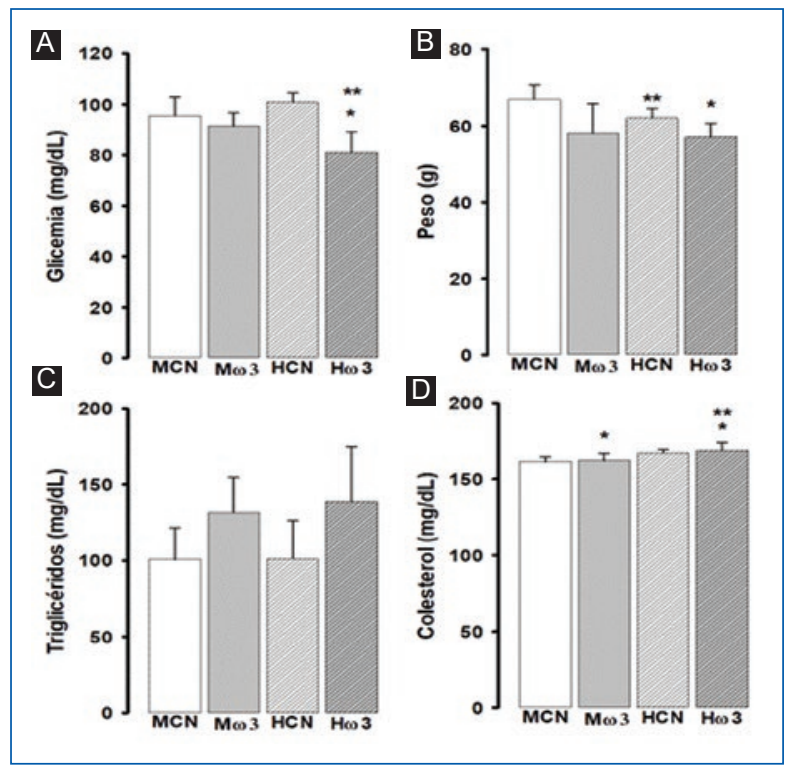

Figura 1. Parámetros fisiológicos de ratas lactantes con suplemento de ácidos grasos omega 3 de cadena corta. A: glicemia; B: peso corporal; C: triglicéridos; D: colesterol. MCN: machos control; M $\omega 3$ : machos con tratamiento; HCN: hembras control; $\mathrm{H} \omega 3$ : hembras con tratamiento. ${ }^{*} p<0.05$ factor tratamiento; ${ }^{* *} p<0.05$ factor sexo $(n=6)$.

la finalidad de causar la eutanasia ${ }^{9} \mathrm{y}$ drenar la sangre de los órganos), y se extrajeron el hígado y el cerebro $^{10}$. Se determinaron los parámetros morfométricos del cerebro (peso, densidad y la proporción cerebro/ masa corporal [Fórmula 1]) y el índice de encefalización (EQ) (Fórmula 2). Se realizó el aislamiento de mitocondrias de tejido hepático como se describe en Figueroa, et al. (2015) ${ }^{11}$. La respiración mitocondrial (consumo de oxígeno) se evaluó en los estados 3 y 4 , y se calculó el control respiratorio (velocidad en estado 3/estado 4) ${ }^{11}$. La composición de ácidos grasos se realizó por cromatografía de gases mediante la técnica descrita previamente por Figueroa, et al. $(2015)^{11}$, previa extracción de los lípidos por el método de Folch, et al. $(1957)^{12}$ y transesterificación mediante la técnica de Morrison, et al. (1964)13,14. Se determinó la concentración de malondialdehído (MDA) por el método de TBARS, es descrito por Cow, et al. (1984) ${ }^{14,15}$.

$$
P C M C=\frac{P C 1}{P C 2} \times 100
$$

Fórmula 1. Proporción cerebro/masa corporal.

PC1: peso del cerebro; PC2: peso corporal.

$$
E Q=\frac{\text { Peso del cerebro }}{0.12(\text { Peso corporal })^{0.67}}
$$

Fórmula 2. Ecuación de Jerison.

\section{Análisis estadístico}

Se realizó el análisis de las medidas de tendencia central: promedio $\bar{x}$ y desviación estándar $\sigma$ de los datos obtenidos para ver su distribución, prueba de Shapiro-Wilk para probar normalidad. Los datos fueron analizados mediante análisis de varianza (ANOVA) de dos factores, seguida de la prueba de Bonferroni, con un intervalo de confianza de $0.95(p<0.05)$ en el programa SigmaPlot 13 (2017).

\section{Resultados}

\section{Parámetros fisiológicos}

Los resultados obtenidos muestran que existe diferencia $(p<0.05)$ en la glicemia para el factor tratamiento entre las hembras y para el factor sexo en los individuos con AGw3CC (Fig. 1 A). También se observó diferencia en el peso (Fig. 1 B) para el factor tratamiento entre las hembras y para el factor sexo en los grupos control. La CTG (Fig. 2) está ligeramente alterada en los grupos con $A G \omega 3 C C$. Se encontró que existe diferencia $(p<0.05)$ en el factor sexo, y el área bajo la curva es mayor en los machos. En los grupos de hembras se observa diferencia $(p<0.05)$ entre tratamientos en la glicemia $(20 \mathrm{mg} / \mathrm{dL}$ menor en $\mathrm{H} \omega 3$ con corrimiento de 30 minutos en el pico).

\section{Función mitocondrial y concentración de malondialdehído}

En el estado 4 se observa diferencia $(p<0.05)$ en el factor sexo, con un mayor consumo de oxígeno $\left(\mathrm{nA}^{\circ} \mathrm{O}_{2} / \mathrm{min} / \mathrm{mg}\right.$ Prot) en $\mathrm{H \omega} 3$ (Fig. $3 \mathrm{~A}$ ). Para el caso del estado 3 (Fig. 3 B) se observa diferencia $(p<0.05)$ en el factor tratamiento entre las hembras y en el factor sexo en los animales con AG $03 \mathrm{CC}$. Existe diferencia $(p<0.05)$ entre tratamiento, con una menor concentración de MDA en las hembras (HCN y Ho3) (Fig. 4).

\section{Parámetros morfométricos del cerebro e índice de encefalización}

En el grupo control se puede observar que el peso del cerebro es mayor $(0.5 \mathrm{~g})$ en las hembras $(p<0.05)$. La 


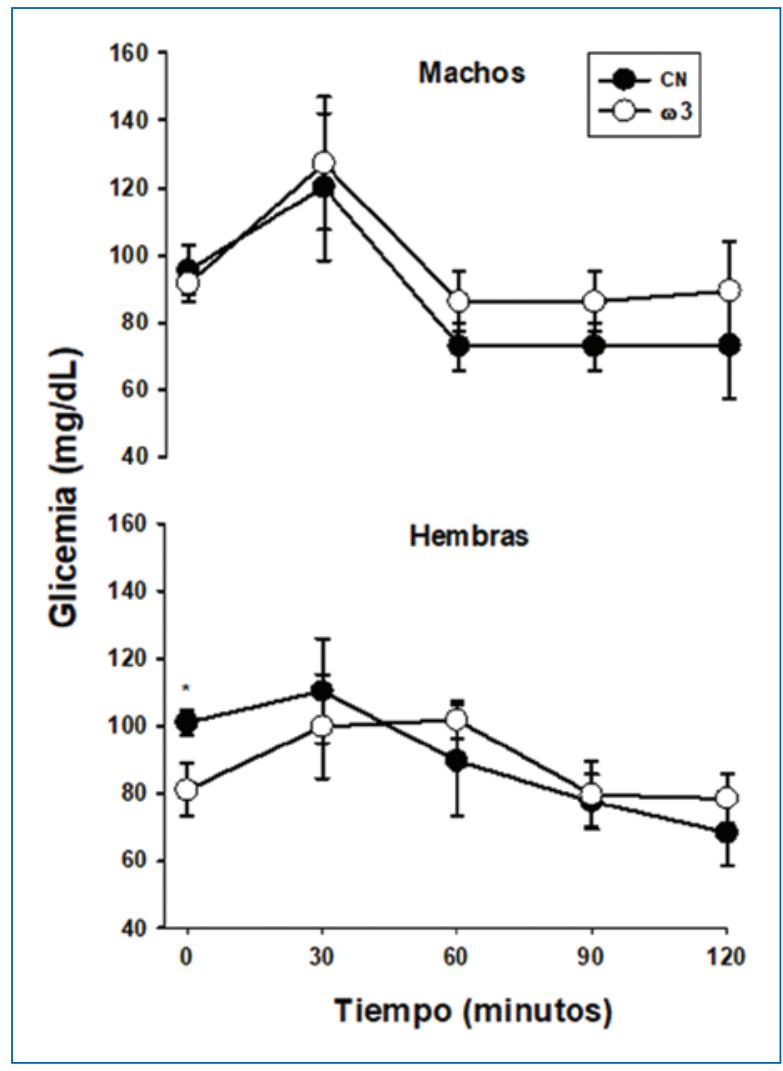

Figura 2. Curvas de tolerancia a la glucosa en ratas lactantes suplementadas con ácidos grasos omega 3 de cadena corta. MCN: machos control; M $\omega 3$ : machos con tratamiento; $\mathrm{HCN}$ : hembras control; $\mathrm{H} \omega 3$ : hembras con tratamiento. ${ }^{*} p<0.05$ factor tratamiento $(n=6)$.

suplementación AGw3CC incrementa el peso del cerebro en los machos $(0.3 \mathrm{~g})$ y lo disminuye en las hembras $(0.1 \mathrm{~g})$, sin cambios aparentes en la densidad del órgano. La concentración de proteína tiene un incremento de 2.5 veces en las hembras con $A G \omega 3 C C(p<0.05)$. La proporción cerebro/masa corporal es mayor $(p<0.05)$ tanto en las hembras como en los machos del grupo $A G \omega 3 C C$ (Tabla 1). El EQ es un parámetro que describe el grado de desarrollo del cerebro y se calcula utilizando la ecuación formulada por Jerison en 1973 (Fórmula 3) ${ }^{16}$. En la figura 5 se observa que el EQ incrementa en los grupos con AG $\omega 3 C C(p<0.05)$, y es mayor en hembras tanto en los grupos control como en los que recibieron $A G \omega 3 C C$.

\section{Composición de ácidos grasos}

Existe diferencia $(p<0.05)$ para la interacción sexo-tratamiento en el tejido hepático (Tabla 2) y la fracción mitocondrial (Tabla 3) de este órgano, con un distinto perfil de ácidos grasos entre machos y
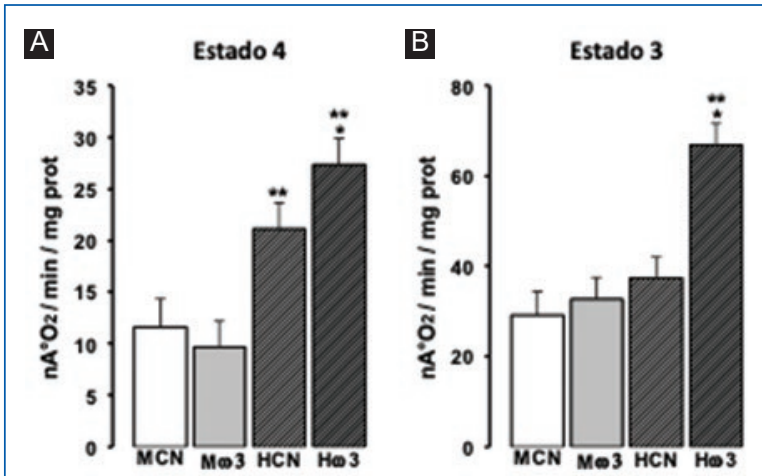

C
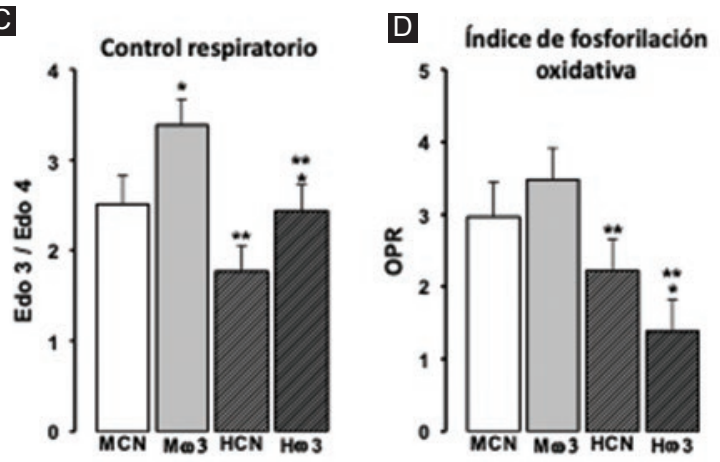

Figura 3. Función mitocondrial (estado $4[A]$ y $3[B]$ ), control respiratorio (C) e índice de fosforilación oxidativa (D) de ratas lactantes suplementadas con ácidos grasos omega 3 de cadena corta. MCN: machos control; M $\omega 3$ : machos con tratamiento; HCN: hembras control; H $\omega 3$ : hembras con tratamiento. ${ }^{*} p<0.05$ factor tratamiento; ${ }^{* *} p<0.05$ factor $\operatorname{sexo}(n=6)$.

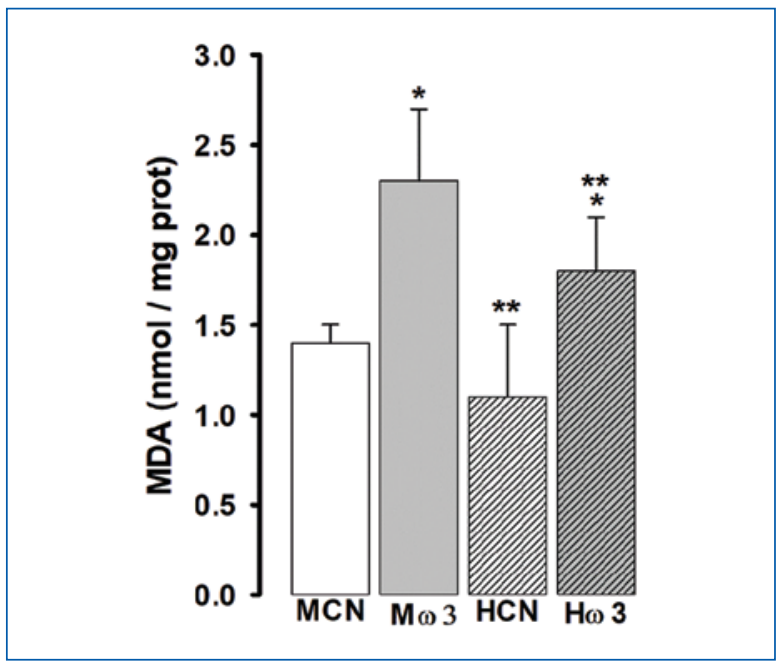

Figura 4. Concentración de malondialdehído (MDA) en mitocondrias de ratas lactantes suplementadas con ácidos grasos omega 3 de cadena corta. MCN: machos control; M $\omega 3$ : machos con tratamiento; HCN: hembras control; H $\omega 3$ : hembras con tratamiento. ${ }^{*} p<0.05$ factor tratamiento; ${ }^{* *} \mathrm{p}<0.05$ factor sexo $(\mathrm{n}=6)$. 
Tabla 1. Parámetros morfométricos del cerebro de ratas lactantes control y suplementadas con ácidos grasos omega 3 de cadena corta

\begin{tabular}{|c|c|c|c|c|}
\hline & MCN & $\mathbf{M \omega 3}$ & HCN & $\mathbf{H} \omega \mathbf{3}$ \\
\hline Peso corporal (g) & $68.400 \pm 4.099$ & $60.000 \pm 7.589$ & $67.500 \pm 5.505$ & $54.167 \pm 5.707$ \\
\hline Peso del cerebro $(\mathrm{g})$ & $1.128 \pm 0.060$ & $1.427 \pm 0.306$ & $1.595 \pm 0.055$ & $1.517 \pm 0.103$ \\
\hline CPC/PC & $0.020 \pm 0.001$ & $0.020 \pm 0.005$ & $0.024 \pm 0.002$ & $0.030 \pm 0.005$ \\
\hline PCMC & $1.650 \pm 0.100$ & $2.400 \pm 0.550$ & $2.380 \pm 0.230$ & $2.840 \pm 0.460$ \\
\hline EO & $0.550 \pm 0.030$ & $0.660 \pm 0.140$ & $0.770 \pm 0.020$ & $0.680 \pm 0.040$ \\
\hline DMC $(\mathrm{g} / \mathrm{mL})$ & $2.200 \pm 0.400$ & $2.330 \pm 0.820$ & $2.000 \pm 0.630$ & $2.500 \pm 0.550$ \\
\hline IDMC/EO & $1.100 \pm 0.200$ & $1.100 \pm 0.400$ & $1.000 \pm 0.300$ & $1.100 \pm 0.200$ \\
\hline Proteína (mg) & $4.220 \pm 1.520$ & $4.720 \pm 0.340$ & $4.110 \pm 2.960$ & $10.640 \pm 1.12$ * \\
\hline
\end{tabular}

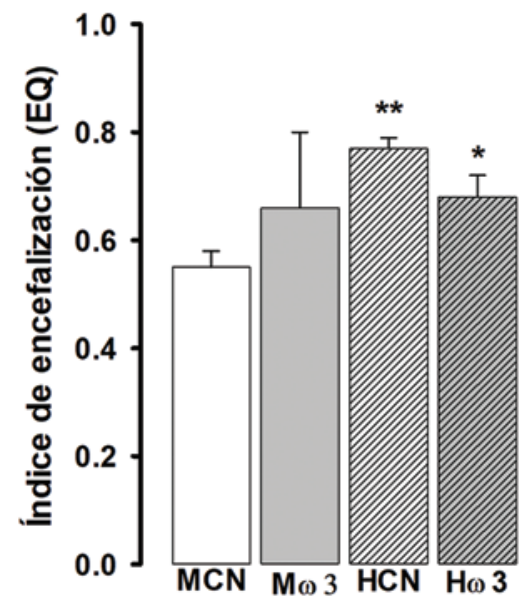

Figura 5. Índice de encefalización de ratas lactantes control y suplementadas con ácidos grasos omega 3 de cadena corta. MCN: machos control; M $\omega 3$ : machos con tratamiento; HCN: hembras control; $\mathrm{H} \omega 3$ : hembras con tratamiento. ${ }^{*} p<0.05$ entre grupos; ${ }^{* *} p<0.05$ entre sexos $(n=6)$.

hembras. En el cerebro (Tabla 4) se encontró que existe diferencia $(p<0.05)$ para el factor sexo. El índice de ácidos grasos insaturados sobre saturados (I/S) muestra diferencia $(p<0.05)$ para el factor sexo y el factor tratamiento en el tejido hepático (Tabla 2) y en el cerebro (Tabla 4).

\section{Discusión}

Existen diversos modelos desarrollados en animales para evaluar los efectos de los $A G \omega 3$ desde diferentes perspectivas, la gran mayoría en machos adultos, en los que factores como el desarrollo, el crecimiento o la etapa fisiológica (hormonal) no influyen sobre las variables de estudio. Sin embargo, es importante señalar que se requieren estudios que aporten información acerca de los efectos, ya sean benéficos o adversos, de estos ácidos grasos en las diferentes etapas de la vida de los individuos, así como la forma en la que estas moléculas afectan a los individuos de acuerdo al sexo. Por ello, el modelo elegido para la realización del presente estudio utiliza ratas de ambos sexos durante la etapa de lactancia. La ventaja de utilizar un modelo donde se incluye tanto a hembras como a machos es la obtención de datos que evidencien si existe una respuesta diferente debido al factor sexo. Por otro lado, el estudio del consumo de $A G \omega 3$ en un modelo de animales lactantes obedece a la realidad moderna, donde las fórmulas lácteas son suplementadas con $A G \omega 3$ o se administran como suplementos alimenticios a infantes, exista o no la necesidad real de hacerlo.

Los resultados del estudio muestran cambios en los parámetros fisiológicos de los animales suplementados con AG $\omega 3 \mathrm{CC}$. Todos ellos asociados a los efectos que tienen estas moléculas en el metabolismo de la glu$\operatorname{cosa}^{17}$ y la síntesis de insulina ${ }^{18}$, por un lado, y su asociación con la pérdida o pobre ganancia de peso, por el otro ${ }^{19}$. Es importante señalar que, además de lo 
Tabla 2. Composición de ácidos grasos del tejido hepático de ratas control y con tratamiento de ácidos grasos omega 3 de cadena corta durante la etapa de lactancia

\begin{tabular}{|c|c|c|c|c|}
\hline \multicolumn{5}{|c|}{ Hígado (tejido) } \\
\hline & MCN & $\mathbf{M} \omega \mathbf{3}$ & HCN & H $\omega 3$ \\
\hline Mirístico & $0.30 \pm 0.07$ & $0.33 \pm 0.09$ & $0.61 \pm 0.11^{* *}$ & $0.65 \pm 0.15^{* * *}$ \\
\hline Miristoleico & $0.19 \pm 0.04$ & $0.46 \pm 0.16^{*}$ & $0.45 \pm 0.19^{* *}$ & $0.67 \pm 0.45^{* *}$ \\
\hline Palmítico & $21.85 \pm 1.35$ & $27.76 \pm 2.72^{*}$ & $20.58 \pm 1.51$ & $23.52 \pm 3.56^{* *}$ \\
\hline Palmitoleico & $0.51 \pm 0.06$ & $0.57 \pm 0.48$ & $1.32 \pm 0.54^{* *}$ & $0.91 \pm 0.35$ \\
\hline Esteárico & $21.31 \pm 1.58$ & $23.11 \pm 1.86$ & $15.36 \pm 3.61^{* *}$ & $17.45 \pm 2.61^{*}$ \\
\hline Oleico & $17.09 \pm 1.07$ & $7.87 \pm 4.20^{*}$ & $24.26 \pm 4.91^{* *}$ & $20.24 \pm 4.70$ \\
\hline Linoleico & $12.90 \pm 1.88$ & $12.89 \pm 1.43$ & $15.45 \pm 1.47^{* *}$ & $14.58 \pm 2.30$ \\
\hline Gama linoleico & $0.03 \pm 0.01$ & $0.03 \pm 0.01$ & & $0.08 \pm 0.05$ \\
\hline Linolénico & $0.07 \pm 0.05$ & $0.13 \pm 0.04$ & $0.33 \pm 0.09 * *$ & $0.60 \pm 0.18^{* * *}$ \\
\hline Araquídico & $0.09 \pm 0.04$ & $0.30 \pm 0.12^{*}$ & $0.09 \pm 0.09^{* *}$ & $0.22 \pm 0.12$ \\
\hline Araquidónico & $14.55 \pm 0.72$ & $14.17 \pm 2.89$ & $11.12 \pm 2.00^{* *}$ & $9.75 \pm 2.62^{* *}$ \\
\hline EPA & $0.41 \pm 0.11$ & $0.51 \pm 0.35$ & $0.71 \pm 0.18^{* *}$ & $1.24 \pm 0.37^{* * *}$ \\
\hline Behenico & $0.17 \pm 0.11$ & $0.21 \pm 0.12$ & $0.23 \pm 0.13$ & $0.28 \pm 0.23$ \\
\hline Erúcico & & $0.04 \pm 0.02$ & $0.03 \pm 0.01$ & $0.19 \pm 0.06^{*}$ \\
\hline Lignocérico & $0.80 \pm 0.05$ & $0.96 \pm 0.13^{*}$ & $0.89 \pm 0.12^{* *}$ & $0.83 \pm 0.25$ \\
\hline DHA & $9.18 \pm 1.50$ & $8.46 \pm 1.65^{*}$ & $6.03 \pm 0.89 * *$ & $5.79 \pm 1.53^{* *}$ \\
\hline $\mathrm{NI}$ & $2.87 \pm 0.28$ & $2.43 \pm 0.44$ & $2.88 \pm 1.14$ & $3.25 \pm 0.56$ \\
\hline Insaturados & $54.94 \pm 7.04$ & $45.25 \pm 5.78$ & $60.17 \pm 10.64$ & $54.06 \pm 12.61$ \\
\hline Saturados & $44.52 \pm 10.26$ & $52.68 \pm 12.18$ & $37.77 \pm 5.56$ & $42.96 \pm 6.93$ \\
\hline $\mathrm{I} / \mathrm{S}$ & $1.23 \pm 0.11$ & $0.86 \pm 0.12^{*}$ & $1.59 \pm 0.34^{* *}$ & $1.26 \pm 0.32^{* * *}$ \\
\hline
\end{tabular}

* $p<0.05$ factor grupo.

${ }^{* *} p<0.05$ factor sexo $(n=6)$.

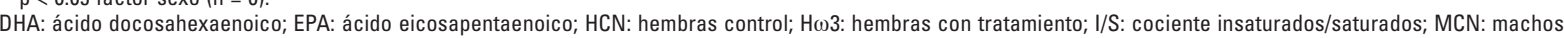
control; Mw3: machos con tratamiento; NI: no identificados.

observado en los animales del grupo suplementado, en los grupos de animales control se encontraron diferencias asociadas al factor sexo. De acuerdo a lo reportado por Pérez, et al. (2011) ${ }^{20}$ y Figueroa $(2013)^{9}$, la glicemia de los grupos control se encuentra en el límite inferior del rango considerado como normal en ratas lactantes (60-101 mg/dl) (Fig. 1 A). Es importante señalar que en los grupos suplementados con AG $\omega 3 C C$ (incluidos machos y hembras) existe una menor glicemia (5-10 mg/dl). Se sabe que los ácidos grasos poliinsaturados (PUFA) están asociados al balance energético, a la utilización de sustratos (lípidos y carbohidratos) y, por lo tanto, a las vías metabólicas involucradas en la homeostasis de la glucosa y metabolismo de lípidos a través de la activación del receptor activado por proliferación de los peroxisomas (PPAR) en las células $\beta$ del páncreas, lo que tiene como efecto el incremento en la síntesis de insulina (hiperinsulinemia) $y$, como consecuencia, un incremento en la utilización de la glucosa en los tejidos periféricos y una disminución de esta molécula en sangre ${ }^{21}$. Esto es de suma importancia, ya que el gasto metabólico de un individuo en crecimiento, y sobre todo en la etapa de lactancia, es elevado, y un deficiente aporte energético pone en riesgo su supervivencia ${ }^{22}$, por lo que el consumo de alimentos de aporte calórico elevado es indispensable para mantener la homeostasis de la glucosa ${ }^{23}$. 
Tabla 3. Composición de ácidos grasos de la fracción mitocondrial de tejido hepático deratas control y con tratamiento de ácidos grasos omega 3 de cadena corta durante la etapa de lactancia

\begin{tabular}{|c|c|c|c|c|}
\hline \multicolumn{5}{|c|}{ Mitocondrias de hígado } \\
\hline & MCN & $\mathbf{M} \omega \mathbf{3}$ & HCN & $H \omega 3$ \\
\hline Mirístico & $0.41 \pm 0.05$ & $0.27 \pm 0.14^{*}$ & $0.23 \pm 0.05^{* *}$ & $0.32 \pm 0.05^{*}$ \\
\hline Miristoleico & $0.01 \pm 0.01$ & $0.05 \pm 0.02 *$ & $0.26 \pm 0.04^{* *}$ & $0.29 \pm 0.09$ \\
\hline Palmítico & $19.28 \pm 1.35$ & $18.45 \pm 1.06$ & $15.75 \pm 1.45^{* *}$ & $19.25 \pm 1.01 * * *$ \\
\hline Palmitoleico & $0.65 \pm 0.28$ & $0.55 \pm 0.33$ & $0.71 \pm 0.26$ & $0.63 \pm 0.21 * * *$ \\
\hline Esteárico & $19.70 \pm 2.00$ & $19.94 \pm 0.34$ & $20.61 \pm 1.00$ & $19.65 \pm 2.28$ \\
\hline Oleico & $11.09 \pm 2.54$ & $11.43 \pm 2.65$ & $13.57 \pm 3.82$ & $11.57 \pm 1.80$ \\
\hline Linoleico & $13.13 \pm 1.88$ & $14.19 \pm 0.76$ & $14.27 \pm 2.04$ & $15.47 \pm 2.40$ \\
\hline Gama linoleico & $0.07 \pm 0.03$ & $0.04 \pm 0.01$ & $0.05 \pm 0.02$ & $0.04 \pm 0.02$ \\
\hline Linolénico & $0.09 \pm 0.04$ & $0.19 \pm 0.05^{*}$ & $0.08 \pm 0.03$ & $0.25 \pm 0.08 * * *$ \\
\hline Araquídico & $0.16 \pm 0.14$ & $0.23 \pm 0.20$ & $0.06 \pm 0.02$ & $0.10 \pm 0.05$ \\
\hline Araquidónico & $16.86 \pm 2.63$ & $16.16 \pm 1.95$ & $16.22 \pm 2.24$ & $15.72 \pm 2.41$ \\
\hline EPA & $0.17 \pm 0.03$ & $0.43 \pm 0.19^{*}$ & $0.35 \pm 0.11$ & $0.90 \pm 0.55^{* * *}$ \\
\hline Behenico & $0.11 \pm 0.05$ & $0.24 \pm 0.13$ & $0.17 \pm 0.07$ & $0.23 \pm 0.08$ \\
\hline Erúcico & $0.07 \pm 0.02$ & $0.08 \pm 0.04$ & $0.05 \pm 0.02$ & $0.08 \pm 0.03$ \\
\hline Lignocérico & $0.90 \pm 0.35$ & $1.22 \pm 0.15$ & $0.87 \pm 0.08$ & $1.00 \pm 0.15$ \\
\hline DHA & $11.63 \pm 2.85$ & $14.03 \pm 1.79$ & $9.90 \pm 0.53^{* *}$ & $10.50 \pm 1.18$ \\
\hline $\mathrm{NI}$ & $5.04 \pm 4.06$ & $2.63 \pm 0.52$ & $3.32 \pm 2.39$ & $3.86 \pm 1.27$ \\
\hline Insaturados & $54.24 \pm 6.68$ & $57.28 \pm 7.06$ & $55.49 \pm 6.87$ & $55.46 \pm 6.88$ \\
\hline Saturados & $40.56 \pm 9.28$ & $40.35 \pm 8.97$ & $37.68 \pm 8.68$ & $40.56 \pm 8.68$ \\
\hline I/S & $1.34 \pm 0.21$ & $1.42 \pm 0.08$ & $1.47 \pm 0.17$ & $1.37 \pm 0.16$ \\
\hline
\end{tabular}

${ }^{*} p<0.05$ factor grupo.

${ }^{* *} p<0.05$ factor sexo $(n=6)$.

DHA: ácido docosahexaenoico; EPA: ácido eicosapentaenoico; HCN: hembras control; Hఱ3: hembras con tratamiento; I/S: cociente insaturados/saturados; MCN: machos

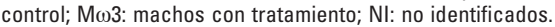

Uno de los mecanismos a través de los cuales los AG $\omega 3$ generan disminución del peso corporal es la regulación del consumo voluntario a través de la sensación de saciedad ${ }^{18}$. Este fenómeno es atribuido a que la ingesta de $A G \omega 3 C C$ aumenta la síntesis y liberación de leptina ${ }^{23}$. Esta saciedad o disminución de ingesta de alimentos podría ser una de las principales causas de disminución de la glucemia en infantes ${ }^{22,24}$, y probablemente la causa de las glucemias observadas en las ratas de los grupos adicionados con $A G \omega 3 C C^{25}$. Por otro lado, la CTG es una prueba útil en el estudio del metabolismo de los carbohidratos o cuando se quiere probar la eficacia de ciertos fármacos como coadyuvantes en la absorción de la glucosa 0 en el estudio de los efectos de ciertos nutracéuticos (suplementos o complementos) o alimentos funcionales sobre la glicemia ${ }^{26}$. Se fundamenta en la pérdida del control de la capacidad para la utilización de la glucosa y se caracteriza por el fallo en el transporte de la glucosa hacia el interior de las células, sobre todo en aquellas que requieren de insulina, lo que ocasiona que la glicemia se mantenga por encima del valor considerado como normal durante periodos de tiempo prolongados $^{27}$. En las diferentes líneas de investigación desarrolladas por Mejía-Zepeda, et al. (2006-2018) $)^{911,14,20}$ en animales diabéticos tipo 2 (DM2) suplementados con $A G \omega 3$ (en diversos esquemas y periodos de administración), se ha observado que la administración crónica de $A G \omega 3$ genera estados de hiperglicemia compatibles con 
Tabla 4. Composición de ácidos grasos del tejido de cerebral de ratas control y con tratamiento de ácidos grasos omega 3 de cadena corta durante la etapa de lactancia

\begin{tabular}{|c|c|c|c|c|}
\hline \multicolumn{5}{|c|}{ Cerebro } \\
\hline & MCN & $\mathbf{M} \omega \mathbf{3}$ & HCN & $H \omega 3$ \\
\hline Mirístico & $0.35 \pm 0.01$ & $0.45 \pm 0.06^{*}$ & $0.35 \pm 0.04$ & $0.33 \pm 0.11^{* *}$ \\
\hline Miristoleico & $0.07 \pm 0.02$ & $0.08 \pm 0.02$ & $0.03 \pm 0.03$ & $0.12 \pm 0.10$ \\
\hline Palmítico & $29.17 \pm 0.94$ & $28.59 \pm 1.10$ & $29.61 \pm 1.75$ & $26.93 \pm 2.51$ \\
\hline Palmitoleico & $0.82 \pm 0.25$ & $0.46 \pm 0.25$ & $0.86 \pm 0.26$ & $0.83 \pm 0.36$ \\
\hline Esteárico & $17.55 \pm 2.68$ & $17.77 \pm 1.53$ & $16.90 \pm 1.65$ & $15.84 \pm 1.60$ \\
\hline Oleico & $18.32 \pm 0.80$ & $20.27 \pm 3.77$ & $19.35 \pm 2.19$ & $20.75 \pm 3.21$ \\
\hline Linoleico & $0.67 \pm 0.26$ & $0.78 \pm 0.09$ & $0.74 \pm 0.12$ & $0.78 \pm 0.15$ \\
\hline Gama linoleico & $0.08 \pm 0.00$ & $0.03 \pm 0.01$ & $0.04 \pm 0.02$ & \\
\hline Linolénico & $0.10 \pm 0.02$ & $0.18 \pm 0.00 * 1$ & $0.18 \pm 0.07^{* *}$ & \\
\hline Araquídico & $0.89 \pm 0.64$ & $0.39 \pm 0.16$ & $0.67 \pm 0.24$ & \\
\hline Araquidónico & $10.13 \pm 2.00$ & $10.38 \pm 1.24$ & $11.58 \pm 1.73$ & $13.68 \pm 1.62^{* * *}$ \\
\hline EPA & $0.91 \pm 0.62$ & $0.35 \pm 0.29$ & $0.47 \pm 0.23$ & $0.42 \pm 0.10$ \\
\hline Behenico & $0.33 \pm 0.11$ & $0.20 \pm 0.07$ & $0.37 \pm 0.10$ & $0.21 \pm 0.08^{* * *}$ \\
\hline Erúcico & $0.49 \pm 0.17$ & $0.17 \pm 0.05^{*}$ & $0.10 \pm 0.07^{* * *}$ & $0.19 \pm 0.13^{* *}$ \\
\hline DHA & $10.52 \pm 2.58$ & $11.92 \pm 1.70$ & $12.76 \pm 2.89$ & $15.04 \pm 2.53$ \\
\hline $\mathrm{NI}$ & $9.58 \pm 2.63$ & $8.63 \pm 1.62$ & $7.46 \pm 3.09$ & $7.47 \pm 1.85$ \\
\hline Insaturados & $42.88 \pm 7.44$ & $44.60 \pm 7.42$ & $46.11 \pm 7.28$ & $53.65 \pm 8.34$ \\
\hline Saturados & $48.22 \pm 4.54$ & $47.40 \pm 2.93$ & $47.90 \pm 12.46$ & $43.31 \pm 11.62$ \\
\hline I/S & $0.89 \pm 0.13$ & $0.94 \pm 0.07$ & $0.96 \pm 0.13^{* *}$ & $1.24 \pm 0.12^{* * *}$ \\
\hline
\end{tabular}

intolerancia a la glucosa, síndrome de resistencia a la insulina y posiblemente DM2 en individuos sanos, por lo que un estudio completo del efecto de estas moléculas en animales en etapa de lactación siempre debe incluir pruebas que evalúen la homeostasis de la glucosa. Es por ello que en el presente trabajo se utilizó la CTG como método de valoración de los posibles cambios metabólicos ocasionados por la administración de AG $\omega 3$ durante la lactancia, y se encontró que el área bajo la curva, calculada mediante la prueba de los polígonos, en los animales del grupo control es el 3.2\% mayor en las hembras; sin embargo, este efecto cambia cuando se administran los $A G \omega 3 C C$, y el resultado es un $8.6 \%$ mayor en los machos. Por otro lado, en el análisis de los TG y el COL no se observaron cambios significativos en los animales con AGw3CC. Estos resultados son similares a los encontrados por De-Lorgeril, et al. (1994) ${ }^{28}$ y Ferrier, et al. (1995) ${ }^{29}$. Es importante señalar que en la concentración de TG se observa un ligero cambio en los animales de los grupos $A G \omega 3 C C$, el cual se puede explicar por la movilización de los ácidos grasos para la obtención de energía, y se correlaciona en forma positiva $(R=0.99)$ con la pobre ganancia de peso (12\% en machos y $19.7 \%$ en hembras) (Figs. $1 \mathrm{C}$ y $1 \mathrm{D}$ ), valores considerados como un índice de desnutrición leve (machos, 0.86 y hembras, 0.91) que indica un estado catabólico debido al gasto energético. Se sabe que durante los estados de estrés, para la conservación de la homeostasis, el organismo es capaz de implementar ciclos de retroalimentación negativa que conduzcan a un estado de equilibrio entre la 
degradación y el uso de los productos energéticos ${ }^{30}$. Esto es, pese a que hay un mayor aporte de productos energéticos (ALA), los cuales son energéticamente menos valiosos que los ácidos grasos saturados, el organismo no puede echar mano de ellos debido a la retroalimentación negativa de la hormona insulina, mecanismo derivado de la estimulación constante de los PPAR $\gamma$ (gamma) en las células beta pancreáticas, lo que a su vez provoca el incremento de glucagón, el cual estimula la movilización de las reservas energéticas del hígado y la lipólisis en el tejido adiposo ${ }^{31,32}$. Por otro lado, el análisis de la concentración sanguínea de colesterol no muestra diferencia estadística entre grupos, lo cual es contrario a otras investigaciones donde se observa una disminución de esta molécula en sangre y otros tejidos en individuos que consumieron dietas enriquecidas con $\mathrm{ALA}^{33}$. Este fenómeno probablemente se deba a que las ratas lactantes no sintetizan hormonas esteroideas en la misma cantidad que una rata adulta, por lo que la cantidad de colesterol no cambia. La concentración de hormonas se controla por su tasa de síntesis, lo que a su vez se rige por señales cerebrales, manteniendo así concentraciones similares en las ratas lactantes sin importar su sexo ${ }^{34}$.

El estudio de la composición de ácidos grasos tanto en tejido como en extractos de mitocondrias de hígado orienta acerca de los cambios en el metabolismo, no únicamente de este órgano sino del sistema en su conjunto, ya que de su buen funcionamiento depende la economía energética del sistema. Es por ello que uno de los primeros cambios que se observan en individuos con problemas metabólicos es la alteración de los parámetros bioquímicos de este órgano ${ }^{35}$. Los principales cambios en la composición de los ácidos grasos se observan en el perfil mitocondrial de hígado de los individuos que recibieron $A G \omega 3 C C$, con un aumento en la concentración de ALA y ácido eicosapentaenoico (EPA) (Tabla 3), lo que se explica por el efecto de competencia entre las enzimas elongasas (Elov-2 y Elov-5) y desaturasas $(\Delta-5$ y $\Delta-6)$ entre ALA y ácido linoleico (AL), como se describe en otras investigaciones ${ }^{36,37}$. Por otra parte, en los tejidos (Tabla 2), se encontró un incremento en la concentración de ALA (81.81\%) y EPA (74.64\%) únicamente en $\mathrm{H} \omega 3$, así como una disminución de los ácidos grasos insaturados (30\%) en los $\mathrm{M} \omega 3$, lo que hace que el I/S sea menor. El cerebro está compuesto esencialmente por lípidos ${ }^{38}$, de los cuales los PUFA son de suma importancia para el desarrollo anatómico y cognitivo del órgano. La composición de los ácidos grasos puede variar en el tejido cefálico dependiendo de la edad (aumenta durante el desarrollo y disminuye con el envejecimiento), el estado de salud (nutrición) y la dieta de los individuos. La composición de ácidos grasos en el cerebro (Tabla 4) varía entre los animales que recibieron ALA durante la lactancia y los animales del grupo control. Al analizar los PUFA, se encontró que el más abundante es el ácido docosahexaenoico (DHA). Este ácido también ha sido reportado como el más abundante en mamíferos ${ }^{39}$. Algunos autores señalan que tanto las células de la glía como las epiteliales tienen la capacidad de sintetizarlo a partir del ALA y otros precursores $^{40}$. Además de lo anterior, existe una menor concentración de EPA en los animales que recibieron ALA $(61.5 \%$, machos y $10.63 \%$, hembras) y un incremento $(25 \%)$ del ácido araquidónico en las hembras de ambos grupos. Es importante señalar que estos ácidos grasos, además de estar implicados en los procesos de inflamación, son componentes estructurales de las membranas celulares, y cualquier cambio en su concentración puede modificar las características físico-químicas de estas estructuras, como la fluidez membranal, la cual puede ser inferida a partir del I/S, por lo que suponemos que existe una mayor fluidez membranal en los grupos tratados con ALA (0.89; machos y 1.24; hembras).

Partiendo de la hipótesis de que los PUFA pueden modificar la función y composición de las membranas mitocondriales, Barzanti, et al. (1994) ${ }^{41}$ encontraron que una dieta rica en omega 3 y 6 modifica la composición de ácidos grasos de mitocondrias de hígado, corazón y cerebro, así como la actividad de ciertas enzimas de interés energético y de transporte, como los complejos citocromo y la ATPasa (bomba sodio-potasio), y llegaron a la conclusión de que la modificación en la actividad de estas y la concentración de ácidos grasos insaturados y saturados puede influir en la homeostasis estructural del organelo. En el presente trabajo se observaron cambios en la concentración de ALA y EPA, lo que concuerda con la literatura, en la cual se menciona que una dieta rica en omega 3 provee los suficientes ácidos grasos de esta familia para mantener concentraciones constantes de los mismos en los tejidos y membranas mitocondriales ${ }^{42}$. De igual forma, los posibles efectos de estas modificaciones en los ácidos grasos de las membranas sobre las proteínas y enzimas mitocondriales podrían explicarse de manera parcial con los resultados observados en las pruebas de respiración mitocondrial (Fig. 3) y lipoperoxidación (Fig. 4), ya que en los tratamiento con AG $\omega 3 C C$ se observó al mismo tiempo el aumento de la concentración de MDA y una tendencia al aumento de la respiración mitocondrial tanto en machos como en hembras. En esta investigación, el I/S no muestra 
diferencias significativas, y las diferencias puntuales de ciertos ácidos grasos no parecen afectar la composición total de esta estructura. Es interesante ver el aumento coordinado de dos parámetros: lipoperoxidación y una correcta respiración mitocondrial. Se ha determinado que aun cuando existe una gran cantidad de especies reactivas de oxígeno, producto de una dieta rica en $A G \omega 3$, esto no afecta las funciones de las mitocondrias, por lo que puede decirse que las membranas mitocondriales no están siendo dañadas por estos y, por ende, la respiración mitocondrial se lleva a cabo sin contratiempos ${ }^{42}$.

De alguna manera, el estudio en conjunto de los parámetros antes mencionados está relacionado directamente con el desarrollo del cerebro y su capacidad cognitiva. Es importante señalar que uno de los eventos más importantes involucrados en la evolución del ser humano es justamente el desarrollo del cerebro (incremento de masa cefálica), el cual está directamente asociado a la cantidad (número) de neuronas que posee, y esta a «la facultad de la mente que permite aprender, entender, razonar, tomar decisiones y formarse una idea determinada de la realidad" (RAE, $2017)^{43}$. Eventos que en su conjunto se definen como inteligencia. En el presente estudio se puede observar que el peso del cerebro en machos del grupo adicionado con ALA es mayor (27.2\%) al del grupo control, circunstancia que se puede considerar como capacitante para tener un mayor desarrollo intelectual. Por otro lado, existe una diferencia de esta variable entre sexos: el peso de este órgano es mayor (31.2\%) en las hembras. En las ratas, parámetros de tipo físico-químico, como la densidad, podrían explicar el incremento de la masa cefálica, lo que modificaría de forma sustancial los resultados y su interpretación.

La medición de algunos parámetros morfométricos del cerebro ayuda a comprender el desarrollo anatómico de este órgano y se puede emplear para explicar algunos cambios en la conducta y cognición de los individuos: en el presente estudio se evaluaron el peso, la densidad y la concentración de proteína (Tabla 1). A partir de estos parámetros se puede calcular el índice de encefalización (Fig. 5), que es el parámetro empleado para medir el grado de desarrollo cognitivo de los individuos.

Una de las principales razones por las cuales se realizó el presente estudio es la gran cantidad de información encontrada, no solo científica sino también comercial, acerca de las propiedades benéficas que tienen los $A G \omega 3$ en el desarrollo cognitivo de infantes ${ }^{44}$, su participación en el tratamiento de enfermedades psiquiátricas (esquizofrenia) ${ }^{45}$, enfermedades degenerativas del sistema nervioso central (Parkinson, Alzheimer, epilepsia) ${ }^{45}$ y trastornos de la conducta (hiperactividad, déficit de atención o ansiedad) (Tapia, 2005) ${ }^{46,47}$.

Es importante señalar que los animales del presente estudio son considerados como animales totalmente sanos, lo cual implica que no presentan ninguna enfermedad o trastorno, por lo que únicamente se estimaron aquellos parámetros que tienen que ver con el aumento en las capacidades cognitivas o inteligencia evaluados a través de criterios anatómicos, como son la proporción cerebro/masa corporal y el cociente de encefalización ${ }^{48}$.

Los resultados obtenidos de la variable proporción cerebro/masa corporal (PC/MC) muestran un menor índice en los animales de los grupos control: $45.5 \%$ (machos) y $19.3 \%$ (hembras) $(p<0.05)$. Es importante señalar que el PC/MC es menor en los machos (44.2\%, controles y $18.3 \%$, suplementados con omega 3 ) en comparación con las hembras. Esto sugiere que el desarrollo del cerebro es mayor en los animales que recibieron ALA y que el cerebro de las hembras se desarrolla con mayor rapidez que el de los machos. Es posible que este simple hallazgo haga suponer que los animales suplementados con omega 3 tendrán una mejor respuesta cognitiva que aquellos que no fueron adicionados; sin embargo, es importante señalar que existen otros parámetros que se pueden utilizar con el fin de dilucidar esta hipótesis. En este caso, la densidad del cerebro es uno de los parámetros que se emplearon para saber si en realidad la PC/MC se debe efectivamente a un incremento en la masa cefálica o es un fenómeno derivado de algún otro factor no considerado en el estudio.

Como ya se vio anteriormente, el peso del cerebro es mayor en los grupos suplementados con ALA; sin embargo, la densidad es menor (5.9\%, machos y $25 \%$, hembras), y existe una diferencia de esta variable entre sexos: la densidad es mayor (56.8\%) en las hembras (0.88 D). Recordemos que la densidad es la relación existente entre la masa y el volumen de un cuerpo, por lo que esta depende de los componentes tanto sólidos como líquidos del mismo. Una característica importante del cerebro es la gran cantidad de lípidos que contiene, y que han sido estudiados como la posible causa de las capacidades cognitivas de los seres humanos ${ }^{49}$. Una de las hipótesis que se estudian desde hace ya mucho tiempo respecto a la inteligencia, en relación con los ácidos grasos que componen el cerebro, es la capacidad de mielinización, ya que de esta característica depende la velocidad de la 
conducción nerviosa, variable asociada a la capacidad de respuesta ${ }^{50}$. De acuerdo a Crawford, et al. (1992) $)^{51}$, la capacidad sináptica entre neuronas genera el desarrollo de complejos patrones celulares (hoy conocidos como mapas mentales). Esto a través de la transferencia de la información de las terminaciones nerviosas sensoriales (gracias a las vainas de mielina en los axones) de las células nerviosas, las que a su vez trasmiten la información a otras neuronas ${ }^{51}$. Sin embargo, el desarrollo de estos mapas mentales, los cuales se han asociado al tamaño del cerebro, no depende únicamente de la eficiente transmisión del impulso nervioso o la comunicación entre las neuronas, sino de la densidad de neuronas (número de neuronas) y el número de uniones entre ellas (sinapsis). Esto explica la gran inteligencia de cerebros pequeños, por lo que el tamaño del cerebro cobra importancia solo si el número de neuronas y uniones sinápticas es equivalente.

Por otra parte, el estudio del índice de encefalización muestra que, a pesar de que los grupos adicionados con omega 3 tienen una mejor proporción cerebro/ masa corporal, esta no está en relación con el grado de inteligencia, ya que los resultados obtenidos sugieren que solo en los machos se correlacionan estas variables $(R=0.89)$, con un $E Q$ el $20 \%$ menor en el grupo control, mientras que en las hembras el grado de asociación es muy bajo $(R=0.68)$, con un $E Q$ un $11.7 \%$ mayor en los animales del grupo control (Tabla 1 ). Además de lo anterior, al comparar los resultados entre machos y hembras se encontró que las hembras tanto del grupo control (40\%) como del grupo con omega $3(3 \%)$ tienen un mejor EQ que los machos ${ }^{52}$.

Una de las características del cerebro de la rata es su rápido crecimiento durante la lactancia, donde hay una sustancial síntesis de ARN, ADN y proteínas ${ }^{53}$, lo que explicaría el incremento en la densidad de los cerebros de los animales del grupo adicionado con AG $\omega 3$. Uno de los efectos de los omega 3 es la estimulación de la insulina, la cual participa, no solo en la incorporación de la glucosa en el tejido adiposo y el muscular, sino también en la proliferación celular ${ }^{54}$.

La información acerca de los efectos de los AG 03 en infantes (humanos y ratas) está enfocada a la evaluación de los beneficios que estas moléculas tienen en el desarrollo cognitivo; sin embargo, gran parte de esta información se enfoca en la estimación de parámetros subjetivos, como habilidad y destreza ${ }^{55}$. En ninguno de los documentos científicos y/o académicos revisados se aborda el análisis fisiológico o bioquímico de las diferencias encontradas en los individuos de estudio y sus posibles efectos en el desarrollo del cerebro y/o cognición. En el presente estudio se evalúan algunos parámetros bioquímicos y físico-químicos relacionados con la fluidez de las membranas y la producción de energía, los cuales afectan directamente la transmisión del impulso nervioso y, con ello, el desarrollo anatómico-cognitivo de los sujetos, como son: composición de ácidos grasos, control respiratorio mitocondrial y lipoperoxidación.

\section{Conclusión}

La administración crónica de AG $\omega 3 \mathrm{CC}$ en ratas lactantes tiene efectos puntuales en los parámetros morfológicos (peso corporal y peso de los órganos) y fisiológicos (concentración de glucosa, triglicéridos y colesterol), modifica la concentración de ciertos ácidos grasos sin que esto tenga aparentemente efectos sustanciales en la composición general de estas moléculas en mitocondrias y/o tejido hepático, modifica la producción de especies reactivas de oxígeno y el cociente respiratorio. Existe un efecto matemático de una diferencia tanto en la composición de ácidos grasos de hígado como en el tejido cerebral entre hembras y machos en la etapa de lactancia cuando son suplementados con AGw3CC.

\section{Conflicto de intereses}

Los autores declaran no tener conflicto de intereses alguno.

\section{Financiamiento}

Este trabajo fue financiado por el Proyecto PAPIIT IN-215917.

\section{Responsabilidades éticas}

Protección de personas y animales. Los autores declaran que los procedimientos seguidos se conformaron a las normas éticas del comité de experimentación humana responsable y de acuerdo con la Asociación Médica Mundial y la Declaración de Helsinki.

Confidencialidad de los datos. Los autores declaran que en este artículo no aparecen datos de pacientes.

Derecho a la privacidad y consentimiento informado. Los autores declaran que en este artículo no aparecen datos de pacientes. 


\section{Bibliografía}

1. Simopoulos AP. Evolutionary Aspects of Diet: The Omega-6/Omega-3 Ratio and the Brain. Molecular Neurobiolog. 2011;44:203-15.

2. Valenzuela BA, Valenzuela R, Sanhueza J, Morales G. Alimentos funcionales, nutraceúticos y foshu: ¿vamos hacia un nuevo concepto de alimentación? Rev Chil Nut. 2014;41(2):198-204.

3. Biruete $A$, Juárez $E$, Sierio $E$, Romero R, Silencio JL. Los nutracéuticos. Lo que conviene saber. Rev Mex Ped. 2009;76(3):136-45.

4. Innis MS. Dietary (n-3) Fatty Acids and Brain Development. J Nutr. 2007:137:855-9

5. Aguirre MP. Acción del omega 3 en el embarazo y la lactancia. [Tesis de licenciatura]. Ciudad de México: Universidad Nacional Autónoma de México; 2008

6. Kim KB, Nam YA, Kim HS, Hayes AW, Lee BM. 凶-Linolenic acid: Nutraceutical, pharmacological And Toxicological Evaluation. Food Chem Toxicol. 2014;70:163-78.

7. Whishaw IQ, Kolb B. The behavior of the laboratory rat: A handbook with tests. Oxford University Press; 2005

8. Norma Oficial Mexicana NOM 062-ZOO-1999. Especificaciones técnicas para la producción, cuidado y uso de los animales de laboratorio. SENASICA. Ciudad de México, México; 6 de diciembre de 1999.

9. Figueroa García MC, Mejía Zepeda R, dir. Análisis de las Alteraciones Morfologicas y Funcionales por Peroxidación de Lípidos de la Membrana Mitocondrial de la Placenta en Ratas Gestantes Inducidas a Diabetes Mellitus Tipo 2 con STZ. [Tesis de doctoral]. Ciudad de México: Universidad Nacional Autónoma de México; 2013.

10. American Veterinary Medical Association. AVMA Guidelines on Euthanasia (Formerly Report of the AVMA Panel on Euthanasia). AVMA; 2007. p. 1-39.

11. Figueroa-García MC, Espinosa-García MT, Martínez-Montes F, Palomar-Morales M, Mejía-Zapeda R. Even a chronic mild hyperglycemia affects membrane fluidity and lipoperoxidation in placental mitocondria in Wistar rats. PLoS One. 2015; 10 (12), e0143778.

12. Folch J, Lee M, Stanley S. A simple method for the isolation and purification of total lipids from animal tissues. J Biol Chem. 1975;226:497-509.

13. Morrison WR, Smith LM. Preparation of fatty acid methyl esters and dimethylacetals from lipids with boron fluoride-methanol. Lipid Res. 1964;5:600-8

14. Perusquía García R, Mejía Zepeda R, dir. Composición de ácidos grasos de mitocondrias hepáticas en diabetes mellitus tipo-2: efecto de ácidos grasos omega-3. [Tesis de licenciatura]. Ciudad de México: Universidad Nacional Autónoma de México; 2008.

15. Cow PB, Gross RW, Sobel BE. Amphipathic metabolites and membrane dysfunction in ischemic myocardium. Circ Res. 1984;55:135-54

16. Jerison JJ. Animal intelligence as encephalization Indice encefalización. Phil Trans R Soc Lond B. 1985;308:21-35.

17. Bajaj M, Suraamornkul S, Hardies LJ, Glass L, Musi N, DeFronzo RA. Effects of peroxisome proliferator-activated receptor (PPAR)- $\square$ and PPAR- $\gamma$ agonists on glucose and lipid metabolism in patients with type 2 diabetes mellitus. Diabetologia. 2007:50(18):1723-31.

18. Ye JM, Doyle PJ, Iglesias MA, Watson DG, Cooney GJ, Kraegen EW Peroxisome proliferator-activated receptor (PPAR)-alpha activation lowers muscle lipids and improves insulin sensitivity in high fat-fed rats: comparison with PPAR-gamma activation. Diabetes. 2001;50:411-7.

19. Parra D, Ramel A, Bandarra N, Kiely M, Martínez JA, Thorsdottir I. A die rich in long chain mega-3 fatty acids modulates satiety in overweight and obese volunteers during weight loss. Appetite. 2008;51:676-80.

20. Pérez-Hernández IH, Mejía Zepeda R, dir. Efecto del aceite de linaza (ácido alfa linolénico) sobre la fluidez membranal en células de ratas espontáneamente hipertensas con diabetes. [Tesis de licenciatura]. Ciudad de México: Universidad Nacional Autónoma de México; 2011. p 131.

21. Guerre-Millo M, Gervois P, Raspe E, Madsen L, Poulain P, Derudas B, et al. Peroxisome proliferator-activated receptor alpha activators improve insulin sensitivity and reduce adiposity. J Biol Chem. 2000; 275:16638-42.

22. Kim KB, Nam YA, Kim HS, Hayes AW, Lee BM. 囚-Linoleic acid: Nutraceutical, pharmacological and toxicological evaluation. Food Chem Toxicol. 2014;70:163-78

23. Davis B, Morris T. Physiological Parameters in Laboratory Animals and Humans. Pharmaceutical Research. 1993;10(7):1093-4.

24. Wang $H$, Storlien LH, Huang $X$. Effects of dietary fat types on body fatness, leptin, and ARC leptin receptor, NPY, and AgRP mRNA expression. Am J Physiol Endocrinol Metab. 2002:282:1352-9.

25. Burnol AF, Leturque A, Ferre P, Girard J. Glucose metabolism during lactation in the rat: quantitative and regulatory aspects. Am J Physiol. 1983;245(4):351-8.

26. Wolever TM, Jenkins DJ. The use of the glycemic index in predicting the blood glucose response to mixed meals. Am J Clin Nutr. 1986; 43(1):167-72.
27. Girbés BJ. Métodos para la determinación de la sensibilidad a la insulina basados en la sobrecarga oral de glucosa. Av Diabetol. 2008; 24(4): 296-304.

28. De Lorgeril M, Renaud S, Mamelle N, Martin JL, Monjaud I, Guidollet J, et al. Mediterranean alpha-linolenic acid-rich diet in secondary prevention of coronary heart disease. Lancet. 1994;343:1454-9.

29. Ferrier LK, Caston LJ, Leeson S, Squires J, Weaver BJ, Holub BJ. 囚-Linolenic acid- and docosahexaenoic acid-enriched eggs from hens fed flaxseed: influence on blood lipids and platelet phospholipid fatty acids in humans. Am J Clin Nutr. 1995;62:81-6.

30. Martínez PG, Paredes DN. Sobre la atrofia de los órganos durante la inanición. Nutr Hosp. 2007;22(1):112-23.

31. Calder CP. Mechanism of Action of (n-3) Fatty Acids. J Nutri. 2011; 142:592-9.

32. Janani $C$, Ranitha Kumari BD. PPAR gama gene -A review. Diabetes Metab Syndr: Clin Res Rev. 2015;9:46-50.

33. Garg ML, Clandinin MT. Alpha-linolenic acid and metabolism of cholesterol and long-chain fatty acids. Nutrition. 1992;8(3):208-10.

34. Mathews KC, Van Holde KE, Appling RD, Anthony-Cahill SJ. Bioquímica. 4. ${ }^{a}$ ed. Madrid: Pearson Educación; 2013. p. 1376.

35. Comós JB, Valles MM. Obesidad y síndrome metabólico. Asociación Española de Pediatría. Protocolos diagnósticos y terapéuticos en Pediatría. 2011;1:228-35.

36. Smink W, Gerrits WJJ, Gloaguen M, Ruiter A, van Baal A. Linoleic and a-linolenic acid as precursor and inhibitor for the synthesis of long-chain polyunsaturated fatty acids in liver and brain of growing pigs. Animal. 2012; 6:(2):262-70.

37. Osorio JH. Metabolismo de los lípidos durante el embarazo. Rev Colomb Obstet Ginecol. 2000;51(2):113-7.

38. Leyva-Rendón A. DHA y funcionamiento cerebral: ¿Cuáles son los beneficios? Rev Mex Neurol. 2011;12(6):365-72

39. Crawford MA. The Role of Dietary Fatty Acids in Biology: Their Place in the Evolution of the Human Brain. Nutrition Reviews. 1992;50(4):3-11.

40. Jicha GA, Markesbery WR. Omega-3 fatty acids: potential role in the management of early Alzheimer disease. Clin Intervent Aging. 2010; 5:45-61.

41. Barzanti V, Battino M, Baracca A, Cavazzoni M. The effect of dietary lipid changes on the fatty acid composition and function of liver, heart and brain mitochondria in the rat at different ages. $\mathrm{Br} \mathrm{J}$ Nutr. 1994; 71:193-202.

42. Herbst EAF, Paglialunga S, Gerling C, Whitfield J, Mukai K, Chabowski A, et al. Omega-3 supplementation alters mitochondrial membrane composition and respiration kinetics in human skeletal muscle. J Physiol. 2014;592:1341-52

43. Diccionario de la Lengua Española (RAE). 2019. Recuperado de: http:// dle.rae.es/?id=LqtyoaQ/LqusWqH

44. Coronado HM, Vega y León S, Gutiérrez TR, García Fernández B, Díaz González G. Los ácidos grasos omega-3 y omega-6 nutrición, bioquímica y salud. Facultad de Ciencias de la UNAM. 2006;25(3):72-9.

45. Valenzuela BA, Sanhueza CJ, Nieto KS. El uso de lípidos estructurados en la nutrición: una tecnología que abre nuevas perspectivas en el desarrollo de productos innovadores. Rev Chil Nutr. 2002;29(2):106-15.

46. Tapia SAE. Omega- 3 fatty acids supplementation decreases the aggressiveness, hostility and antisocial behavior. Rev Chil Nutr. 2005;32(2): 95-101.

47. Bazan NG. Neuroprotectin D1-mediated anti-inflammatory and survival signaling in stroke, retinal degenerations, and Alzheimer's disease. J Lipid Res. 2009;50:400-5

48. Boddy AM, Mcgowen MR, Sherwood CC, et al. Comparative analysis of encephalization in mammals reveals relaxed constraints on anthropoid primate and cetacean brain scaling. J Evol Biol. 2012;25:981-94.

49. Bourre JM. Roles of unsaturated fatty acids (especially omega-3 fatty Acids) in the brain at various ages and during ageing. $J$ Nutr, Health Aging. 2004;8(3):163-74

50. Miller EM. Intelligence and brain myelination: A hypothesis. Personality Individual Differences. 1994;17(6):803-32.

51. Crawfor MA. The role of essential fatty acids in neural development: implications for perinatal nutrition. Am J Clin. Nut. 1993;57(Suppl): 793S-710S.

52. Herculano-Houzel S. Encephalization, Neuronal Excess and Neuronal Index in Rodents. Anat Rec. 2007;290:1280-7.

53. Gottlie A, Keydar IK, Epstein HT. Rodent Brain Growth Stages: An Analytical Review. Biol Neonate. 1977:32:166-76.

54. Aizenman Y, De Vellis J. Brain neurons develop in a serum and glial free environment: effects of transferrin, insulin, insulin-like growth factor- I and thyroid hormone on neuronal survival, growth and differentiation. Brain Research. 1986:4(161):32-42.

55. Catalan J, Moriguchi T, Slotnick B, Murthy M, Greiner RS, Salem N. Cognitive defects in docosahexaenoic acid-deficient rats. Behav Neurosci. 2002;116:1022-31. 